\title{
Leptospiral 3-hydroxyacyl-CoA dehydrogenase as an early urinary biomarker of leptospirosis in a Sri Lankan setting - Interim results
}

\author{
AKUI Karunadasa ${ }^{1}$, C Toma ${ }^{2}$, KMPH Senaratne ${ }^{3}$, KGRA Kumara ${ }^{3}$, CD Gamage ${ }^{3}$
}

Introduction and Objectives: The 3-hydroxyacyl-CoA dehydrogenase (3-HADH) enzyme is of leptospiral origin and is excreted with urine of the patient. The objective of this study was to determine whether the 3-HADH enzyme is present in the urine of acute leptospirosis confirmed patients,usingan enzyme-linked immunosorbent assay (ELISA) to assess its likelihood as a screening test for leptospirosis.

Methods:Laboratory confirmation of leptospirosis was done by flaB-nested PCR onplasma samples of acute leptospirosis suspected patients received from the Teaching Hospital Peradeniya. Urine samples of PCR positive patients and the same number of randomly selectedPCR negative patients were subjected to 3-HADH basedELISA.An ELISA plate coated with supernatants of urine and rabbit anti-3-HADH was used as the primary antibody. The absorbance (OD) measurements obtained at $492 \mathrm{~nm}$ were analyzed using SPSS software version 20.0 with descriptive statistics and independent samples t-test.

Results: Forty-one leptospirosis suspected patients were analyzed in this study, of which 31 (75.6\%) were males. The mean fever day for the cohort was 5.12 days, and 17 patients had less than or equal to 4 days of febrile illness. Twelve patients were confirmed positive for leptospirosis byflaB-nested PCR. The OD values for the PCR positive patients ranged from 1.2241 to 0.1530 , whereas those of theselected PCR negative patients were 0.2932 to 0.086 . Urine of PCR positive patients in the early phase of the disease (less than 5 days of fever) contained 3-HDAH enzyme significantly higher levels of enzyme than PCR negative patients in the same phase of illness(t equal variances $)=2.262,95 \%$ CI $(0.014117-0.756312) \mathrm{P}=$ 0.043).

Conclusions: The 3-HADH enzymeexcretedin urinecan be detected by ELISA during the early phase (less than 5 days of fever) of human leptospirosis.

Keywords: Leptospirosis, 3-hydroxyacyl-CoA dehydrogenase, ELISA, urine biomarker

\footnotetext{
${ }^{1}$ Postgraduate Institute of Science, University of Peradeniya, Sri Lanka

${ }^{2}$ Department of Bacteriology, Graduate School of Medicine, University of the Ryukyus, Okinawa 903-0215, Japan

${ }^{3}$ Department of Microbiology, Faculty of Medicine, University of Peradeniya, Sri Lanka
} 